\title{
Conservative Treatment of Dentigerous Cyst Favoring the Normal Eruption of the Involved Teeth
}

\author{
Heena Sarangal ${ }^{1}$, Monika Rohilla ${ }^{2}$, Saumil Mogre ${ }^{3}$, Ritu Namdev ${ }^{4}$
}

\begin{abstract}
Dentigerous cyst is the most common developmental odontogenic cyst of the jaw, which apparently develops by the accumulation of fluid either between the reduced enamel epithelium and enamel or between the layers of the enamel organ. However, the mechanism of development of dentigerous cyst has unlit now remains unclear. Such cyst remains asymptomatic initially unless when infected. Management of dentigerous cyst in children needs special considerations because of the presence of developing permanent tooth buds. The purpose of this case report is to describe the diagnosis of dentigerous cyst in a 10-year-old boy that was developed around an unerupted mandibular first permanent premolar and to describe its management with marsupialization to allow the growing tooth associated with the cyst to continue eruption into the oral cavity.
\end{abstract}

Keywords: Dentigerous cyst, Developmental cyst, Odontogenic cyst

Journal of South Asian Association of Pediatric Dentistry (2019): 10.5005/jp-journals-10077-3016

\section{INTRODUCTION}

Out of the various varieties of cysts, odontogenic developmental cysts are the ones that develop from epithelial remnants of the tooth forming organ and encloses the crowns of an unerupted tooth. Dentigerous cysts account more than $24 \%$ of all the true cyst of the jaw and are the second most common odontogenic cyst after the radicular cyst. Dentigerous cysts are generally discovered accidentally because of the asymptomatic nature of the cyst unless there is an acute inflammatory exacerbation. ${ }^{1}$ Radiographs show a radiolucent, unilocular lesion with well-defined sclerotic margins characterized by the association with a crown of an unerupted tooth. Complications associated with dentigerous cyst include bone deformities, pathological fractures due to large lesions, loss of a permanent tooth, and development of squamous cell carcinoma. ${ }^{2}$ Various treatment options have been mentioned in the literature for the management of dentigerous cyst. Two most common treatment modalities used are the following: (1) enucleation for small lesions and (2) marsupialization for large ones. ${ }^{1,2}$

\section{Case Description}

A 10-year-old boy reported at the Department of Pediatric and Preventive Dentistry with the chief complaint of malaligned teeth. Intraoral examination showed filling with respect to 84 (Fig. 1A). A panoramic radiograph was taken for orthodontic treatment planning, which revealed the presence of unilocular, radiolucent area on the right side of the mandible extending from the distal surface of unerupted permanent canine to the mesial aspect of unerupted permanent second premolar enclosing unerupted permanent first premolar causing displacement of the same (Fig. 2A). The patient's parents were unaware of this as the lesion was asymptomatic. An informed consent was taken. Aspiration biopsy revealed cystic lesion. Marsupialization was chosen as a treatment option due to the large size and involvement of permanent tooth bud. Surgical intervention was performed under local anesthesia. Extraction of 84 and 85 was done to create a large window. After flap reflection, cystic contents were evacuated and irrigation was done to remove any residual fragment.
1,3,4 Department of Pedodontics and Preventive Dentistry, Pandit Bhagwat Dayal Sharma Post Graduate Institute of Medical Sciences, Rohtak, Haryana, India

${ }^{2}$ Department of Pedodontics and Preventive Dentistry, PGIDS, Rohtak, Haryana, India

Corresponding Author: Heena Sarangal, Department of Pedodontics and Preventive Dentistry, Pandit Bhagwat Dayal Sharma Post Graduate Institute of Medical Sciences, Rohtak, Haryana, India, Phone: +91 79733773171, e-mail: sarangalhena91@gmail.com

How to cite this article: Sarangal $H$, Rohilla $M$, et al. Conservative Treatment of Dentigerous Cyst Favoring the Normal Eruption of the Involved Teeth. J South Asian Assoc Pediatr Dent 2019;2(1):22-24.

Source of support: Nil

Conflict of interest: None

The cystic cavity was packed with iodoform gauze. The removed surgical specimen was sent for histopathological examination, which confirmed the diagnosis of the inflammatory dentigerous cyst (Fig. 3). The patient was recalled after every 7 days for dressing change to allow decompression and eruption of permanent teeth. The involved permanent teeth erupted naturally, without any traction forces (Fig. 1B). An orthopantomogram (OPG) was done after a 2-year follow-up, which revealed erupted permanent premolars (Fig. 2B).

\section{Discussion}

The most common type of developmental cyst is the dentigerous cyst with a frequency of 1.44 per 100 unerupted teeth. The most common site of involvement is the molar area in the mandible whereas the anterior region is the most common area in the case of the maxilla. Radiographically, the dentigerous cyst is divided into three types: central, circumferential, and lateral types. ${ }^{3}$ The dentigerous cyst in this case report is of the lateral type.

The etiology of dentigerous cysts is still unclear. There are various theories given in the literature regarding the etiology of dentigerous cysts. The first theory suggests that it develops from the accumulation 


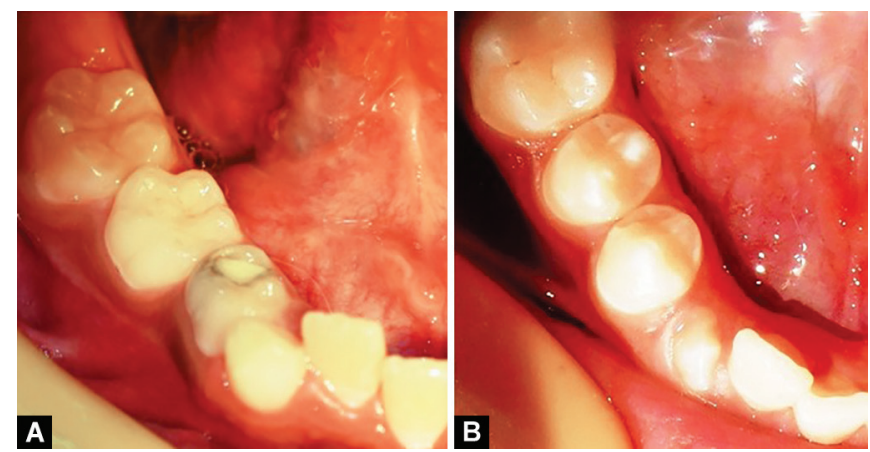

Figs $1 \mathrm{~A}$ and $\mathrm{B}$ : (A) Preoperative clinical picture showing filling with respect to 84; (B) Postoperative follow-up clinical picture showing erupted premolars and canine

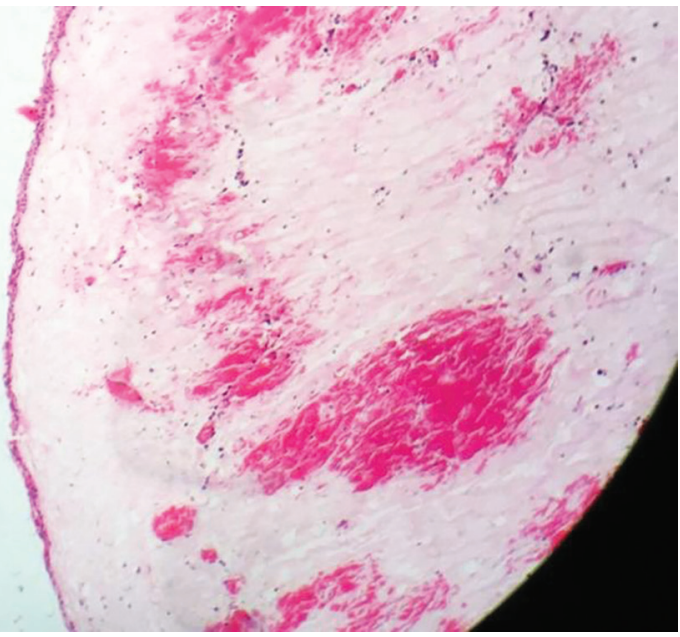

Fig. 3: Histopathological picture of the specimen

fluid between the reduced enamel epithelium and crown of the permanent tooth bud. This is the developmental type of dentigerous cysts and commonly occurs in the late second and third decades. The second theory advocates that the immature permanent tooth encounters a radicular cyst originating from its primary predecessor during eruption. This is the least accepted theory as the occurrence of radicular cyst in association with primary teeth is rare. The third theory describes that the dentigerous cysts are inflammatory in origin and occurs in an unerupted permanent tooth, due to inflammation from a nonvital deciduous predecessor. These types of dentigerous cysts occur most commonly in the first and early part of the second decade. ${ }^{4}$

Differential diagnosis of dentigerous cyst includes hyperplastic follicles, odontogenic keratocyst (OKC), ameloblastoma, ameloblastic fibroma, and eruption cyst. The normal follicular space is $2-3 \mathrm{~mm}$ and a dentigerous cyst can be suspected when the space is more than $5 \mathrm{~mm}$. Dentigerous cysts cause less expansion and less root resorption when compared with OKC. Ameloblastoma shows knife edge root resorption. Radiographically, ameloblastic fibromas mostly do not have tooth structure and calcification within the lesion. Eruption cyst can be differentially diagnosed clinically due to the soft, bluish swelling expanded into the oral cavity. ${ }^{5}$

Radiographic features of dentigerous cysts include unilocular, well-circumscribed, usually symmetric radiolucency larger than that of normal follicular space, enclosing crown of the unerupted permanent tooth. The border is ill-defined, if the cyst is infected. ${ }^{6}$ Histopathological examination revealed thin connective tissue containing islands of the
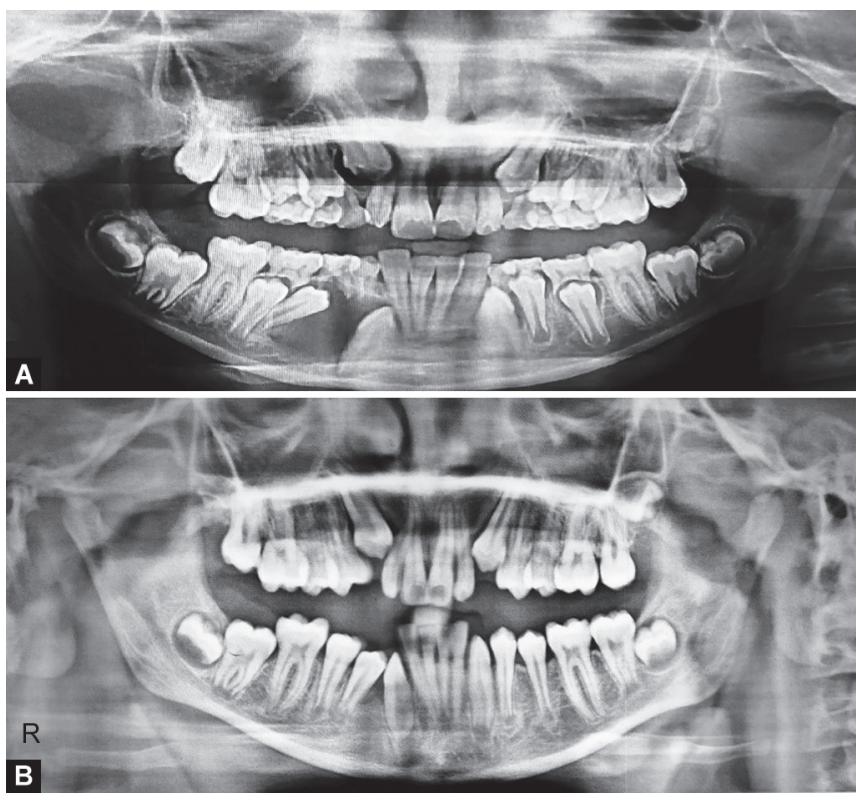

Figs 2A and B: (A) Preoperative OPG showing lesion on the right side of the mandible; (B) Postoperative follow-up OPG showing elimination of lesion and teeth in progressive eruption

odontogenic epithelium with a layer of the squamous epithelium lining the lumen of the cyst. Inflammatory cell infiltration of connective tissue was seen. The presence of Rushton bodies, which are peculiar, hyaline bodies, seen within the epithelium lining. ${ }^{6}$

Various treatment modalities have been described in the literature for the management of dentigerous cyst. The treatment of choice of the lesion depends on the various factors, such as size and location of the cyst, age of the patient, tooth position in relation to the cyst, the degree of axial inclination, relation with the surrounding structures, and affected dentition. ${ }^{4,7}$ Enucleation should be performed when the lesion is small. Marsupialization is a conservative treatment approach in case when the cyst is associated with unerupted permanent tooth germ and important anatomical structures. ${ }^{7}$ In the present case report, marsupialization was the treatment of choice due to the large size of the cyst and its association with the unerupted permanent first premolar.

\section{Conclusion}

As dentigerous cysts are asymptomatic in nature, they can attain considerable large size without the notice of the patient, and this warrants the early clinical and radiographic detection of the cyst, so that early treatment planning will prevent or decrease the morbidity associated with the same. Marsupialization and decompression are very low invasive techniques that could easily be conducted by any dentist familiar with basic surgical procedures, to treat the pathology and preserve the tooth or teeth involved with the cyst.

\section{References}

1. Passi S, Gauba K, et al. Dentigerous cyst in primary dentition: a case report. J Indian Soc Pedod Prev Dent 2008;26(4):168-170. DOI: 10.4103/0970-4388.44035.

2. Hegde RJ, Khare SS, et al. Dentigerous Cyst in a young child: clinical Insight and A Case report. J Indian Soc Pedod Prev Dent 2013;31(3):209-211. DOI: 10.4103/0970-4388.117967.

3. Tilakraj TN, Kiran NK, et al. Non syndromic unilateral dentigerous cyst in a 4 year-old child: a rare case report. Contemp Clin Dent 2011;2(4):398-401. DOI: 10.4103/0976-237X.91813. 
4. Chhabra N, Chhabra A, et al. Conservative management of a dentigerous cyst by marsupialization treatment: clinical insight and a case report. SRM J Res Dent Sci 2018;9(3):148-151. DOI: 10.4103/srmjrds.srmjrds_58_17.

5. Zerrin E, Husniye DK, et al. Dentigerous cysts of the jaws: clinical and radiological findings of 18 cases. J Oral Maxillofac Radiol 2014;2(3): 77-81. DOI: 10.4103/2321-3841.144673.
6. Anjana G, Varma B, et al. Management of dentigerous cyst: a two year review. Int J Clin Pediatr Dent 2011;4(2):147-151. DOI: 10.5005/ jp-journals-10005-1100.

7. Bhardwaj B, Sharma S, et al. Mandibular dentigerous cyst in a10 year old child. Int J Clin Pediatr Dent 2016;9(3):281-284. DOI: 10.5005/ jp-journals-10005-1378. 